\title{
Oxygen Gas in the Arts and Industries
}

\section{By August S. Neumark}

SINCE the discovery of oxygen the civilized world has undergone a revolution in manners and customs. The successful pursuit of innumerable man. ufactures and trades, and the separation of metals from their ores stand in the closest connection with this fact. It may well be stated that the material prosperity of the world has increased many times in this period, and that the fortune of every individual has been augmented in proportion." Thus remarked the distinguished chemist, J. von Liebig, in his "Letters on Chemistry." This was half a century ago. Since then the application of oxygen in the arts and industries has developed to a remarkable degree. OXYGEN IN MEDICINE AND SURGEPY.

The therapeutic use of oxygen dates back to its dis covery. Priestley found that a mouse, confined in limited quantity of oxygen, lived at least twice as long as in a like quantity of atmospheric air. To-day the gas is widely employed in medical practice and surgery. Among the diseases cured or at least re lieved may be mentioned asthma, croup, pneumonia dyspnœa, phthisis (and atomic diseases generally), anæmia, dyspepsia, diabetes, albuminuria, paralysis, and insomnia. The chief method of administration is and insomia. The chief method of administration is sion (intravenous) or introduction into the cavities of sion (intravenous) or introduction into the cavities of
the body are also practised. Oxygen inhalation im. proves the quality of the blood by increasing the red corpuscles. They are of service in the later stages of pneumonia, where there is danger to life from deficient aeration of the blood; further, in chronic bronchitis of old people, and in wasting diseases. Hopeless cases of phthisis have been reported cured. Hopeless cases of phthisis have been reported cured. mucuous membrane, increases the force and velocity of the pulse, and has a quieting effect on the heart and respiration. It shortens the period of convalescence in typhoid fever, is beneficial in chlorosis uræmia, dropsy, diabetic coma, tetanus, and is of dis tinct value in preventing and curing septic complications in gynecology. The introduction of oxygen directly into the intestinal canal may materially aid digestion, and be of service in functional and organic diseases of the liver. Hypodermic injections are use diseases of the liver. Hypodermfe injections are use-
ful in all conditions where the blood is not properly ful in all conditions where the blood is not properly oxidized (sciatica, carbuncles, pleurisy, acute anæmia,
pulmonary tuberculosis). It is often given in conjunction with general anæsthotics, viz., chloroform, ether, ethyl-chloride, and especially with nitrous oxide ("laughing gas"), to prolong anæsthesia, relieving cyanasis and safeguarding against cardiac or respiratory failure. Nitrous oxide mixed with 10 to 15 per cent of oxygen produces perfect anæsthesia withou reducing the anæsthetic power of the former. Ac cording to Dr. G. I. Gwathmey, an anæsthetist of New York, forty thousand administrations of nitrous oxide and oxygen have been recorded without one single fatality. By warming the gases before using them even nausea may be almost completely avoided. Nascent oxygen (i. e., oxygen at the moment of liberation) is one of the most powerful antiseptics known. Peroxide of hydrogen, which readily parts with some o its hydrogen, or oxygenated water (i e., water saturated with oxygen) has distinct value in chronic dyspepsia, vomiting, constipation, and headaches, when taken internally. The peroxides of calcium and magnesium can also be used, as they give up their oxygen when they come in contact with the gastric juice Peroxide of zinc is used as an antiseptic dressing while hydrogen peroxide is one of the best antiseptic washes for wounds, boils, etc. Oxygen baths, i. e. baths in which nascent oxygen is liberated by the action of a catalytic substance on chemicals which contain oxygen loosely combined (such as sodium peroxide or perborate), have beneficial effects on the circulation; cyanasis and dyspnoea has been known to vanish quickly. Oxygen is now much used as a stimulant for athletes. There is no more effective remedy than oxygen gas in cases of asphyxia and in poisoning by exposure to noxious gases such as carbon monoxide, in which the blood looses its oxygencarrying capacity. It is the universal remedy in threatened death from inhaling poisonous gases, such as illuminating gas, benzine vapor, chloroform, ether, ammonia, sulphuretted hydrogen, smoke, sewer gases, acetylene, and "after-damp" of mines. It has further been used in opium, belladonna, chloral, and aniline poisoning, as well as in the resuscitation of the ap parently drowned. The apparatus used for rescue and reviving work will be described elsewhere. The use of oxygen has been suggested for revivifying the atmosphere in public halls. Ozonized air is used in the ventilation of the Chicago Public Library. Ozone is a powerful bactericide and germicide; it purifle the atmosphere, destroys the bacilli of cholera, typhus, anthrax, etc., produces beneficial action when insufflated on wounds, and sterilizes drinking water. It may also be mentioned here that oxygen is used in Rhodesia, S. A., with marked success to combat the deadly South African horse sickness.

Oxygen gas for therapeutic use need not be chemically pure; medically pure oxygen contains between 5 to 10 per cent of nitrogen, according to the method used for preparing it. As long as nitrogen is the only impurity, it can safely be used, but it should not exceed 10 per cent. Oxygen manufactured by the per cent), the impurity being hydrop purity ( 98 to 99 air oxygen is usually of 95 per cent purity, while oxygen prepared by the alkali chlorate process contains anywhere between 5 to 10 per cent nitrogen. If not carefully purified, the gas made by the latter method may be contaminated with chlorine, carbonic acid gas, and traces of solid matter. It may hardly seem plausible, but it is nevertheless a fact, that socalled "medically pure" oxygen, as put up by the manufacturers, is very often unfit for certain technical applications (welding of aluminium, copper, brass), while most of the commercial oxygen sold for welding and like purposes could readily be used for medical purposes, being usually of 95 to 98 per cent purity. And while the latter can be bought for 1 to 2 cents per cubic foot, the cost of the former is as high as

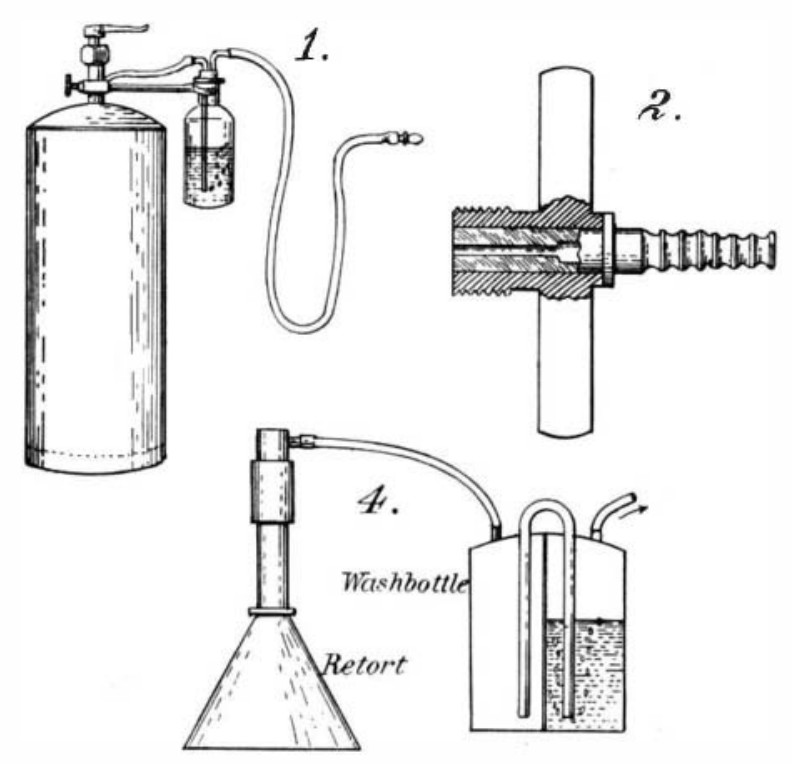

15 cents, or even 20 cents per cubic foot. Medical oxygen is supplied in cylinders (Fig. 1) usually of 100 and 200 gallons' capacity; sometimes smaller tanks holding 50 or 60 gallons are put up. The charging pressure is 225 pounds for any of the sizes mentioned. The cylinders are provided with spindle-valves to which is attached a nozzle (Fig. 2) for the tubing connection. The gas passes from the tank to a wash bottle through which it bubbles, and from there to the mouthpiece or mask. The former should be made of hard rubber as glass is liable to break. High-pressure cylinders should be provided with a reducing valve; from there the oxygen flows through a rubber bag to insure constant pressure. In the low-pressure
tanks the flow of gas can be regulated by the spindle tanks the flow of gas can be regulated by the spindle
valve, the bubbles rising in the water of the wash valve, the bubbles rising in the water of the wash
bottle indicating the force of the current. In most cases the oxygen is inhaled through the mouth, a though in some instances it should be administere through the nose, as in heart failure, pneumonia, and asthma, where the act of respiration is interfered with The gas should be retained in the lungs as long a possible, and should be taken at intervals, and not continuously. In some cases oxygen must be used persistently. The failure to obtain results is often caused by not using the gas freely enough, or by no resorting to it until the patient is in a maribun condition. From four to eight gallons of oxygen can be inhaled without any ill effects, except perhaps a certain nervous exhilaration or a little giddiness; in larger quantities, however, it produces vertigo an narcotism. Compressed oxygen for medical purpose was sold for the first time in New York city by the New York Oxygen Gas Company in 1870, if the write is correctly informed. This firm used the Motay an Marechal process of manufacturing the gas. Befor that time, oxygen was made in the physician's office or at the bedside of the patient, the apparatus conisting of a retort, a wash bottle, and delivery tube the chemicals used were invariably a mixture of chlor- ate of potash and manganese dioxide. (Figs. 3 and 4.) Respiratory devices of various construction are used in the mining and allied industries, in the fire department, in ice and cold storage plants, breweries, and chemical works. Such breathing apparatus are used for rescue work to save life and also to provide against loss of time. In the operation of refrigerating plant accidents occur from time to time, such as the bursting of an ammonia pipe or the breaking of a gage glass, and in such cases respiratory devices are used for rescuing anyone overcome by the deadly fume and for making the necessary repairs without waiting until the entire charge of ammonia is exhausted. The fire department often has occasion to use oxygen. helmets, when the firemen are compelled to enter places filled with smoke and noxious gases. Rescue parties n mine accidents are usually exposed to the dangers incidental to the death-dealing "fire-damp" (marsh gas); therefore emergency breathing devices are kept on hand for instant use. The following incident may be cited to fully illustrate the value of oxygen in gas poisoning: While cleaning a boiler in a German mining plant, six men were overcome by poisonous gases. With no aid in sight, the victims . were left unconscious for nearly an hour, when the foreman remembered having noticed two oxygen tanks in the laboratory. These he carried to the scene of the accident, and then emptied the contents of both cylinders into the boiler. Shortly after, the men began

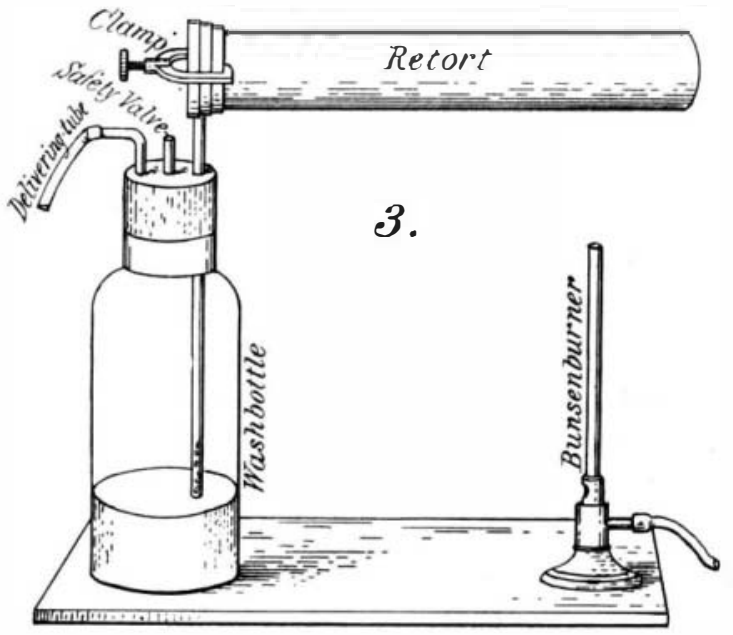

to breathe again, and soon were able to climb un unaided through the manhole.

OXYGEN AS A BLEACH.

Oxygen gas (or ozonized oxygen) in the nascent state or in connection with bleaching powder or chlorine possesses most remarkable bleaching power, its use resulting in considerable economy. It is of special value for bleaching paper pulp, wood, straw, jute, cotton cloth and yarn, also wax, starch, ivory, animal fibers, chamois leather, stearic acid and paraf fin, oils (olive oil, cottonseed oil, linseed oil) rubber goods, etc. If a stream of oxygen is passed through a mixture of paper pulp and a solution of bleaching powder, the decoloration of the pulp proceeds more rapidly than if the bleaching powder alone were used. Oxygen not only accelerates bleaching, but it also reduces the quantity of the powder necessary very considerably. It has been proved by practical test that a saving of 35 to 50 per cent of the bleach can be effected, while air has no such action. Hydrogen peroxide solution is sometimes used for bleaching purposes. Ivory, especially the inferior grade used for knife handles and the like, is bleached that way, also straw, textile and fiber, and white chamois leather. Nascent oxygen or peroxide solution has also been employed for the whitening of the paper of books, prints and engravings that have become stained or dingy with age.

THE USE OF OXYGEN IN THE MATURING OF SPIRITS.

If oxygen under pressure is forced into spirits or liquors and left for about two weeks, the spirit becomes mellowed to an extent equivalent to three to five years aging, nearly all of the fusel oil being converted into harmless compounds. Alcohol and wines can be im proved by either passing oxygen or organized air through the liquid or by adding easily decomposed peroxides. (English Patent No. 18,422 of 1888.) For maturing whisky, oxygen has a very beneficial effect; bad whisky can be transformed into good whisky in a very short time. 
Oxygen is further being used to make solid fish fat, train, and other oils. The last are heated to 90 to 100 deg. C. and then brought into intimate contact with oxygen. It is also used in the production of siccative oils and blown oils for lubricating purpose, a well as for refining linseed and palm oils. A cake of soap made of palm oil refined by this method is said to be remarkable for the delicate fragrance of its odor and its good color. Oxy'gen is also extensively used in the oxidation of oils as required for the manufacture of paints, varnishes, and linoleum. It does away with the use of metallic oxides and exposure to atmospheric air, and the reaction can be completed at (o below) the temperature of boiling water in from three to six hours, while before much higher temperature had to be used for a longer period. There also was the danger from fire in boiling the oil.

The oxidation of linseed oil is effected by passin a current of oxygen in a finely divided stream throug the oil (mixed with a small percentage of a drier) placed in a closed vessel, provided with steam jacket and agitator. As soon as the maximum amount of oxygen is absorbed, it is allowed to cool down to a yellow, jelly-like mass which is solid and dry enough to be packed into bags. This forms the chief ingredient of linoleum. It is mixed with rosin and groun cork, rolled on a jute canvas backing and finally seasoned at a temperature of $75 \mathrm{deg}$. C., whereupon it is ready for use.

Oxygen is being used for the preparation of pure starch derivatives, such as refined starch, soluble
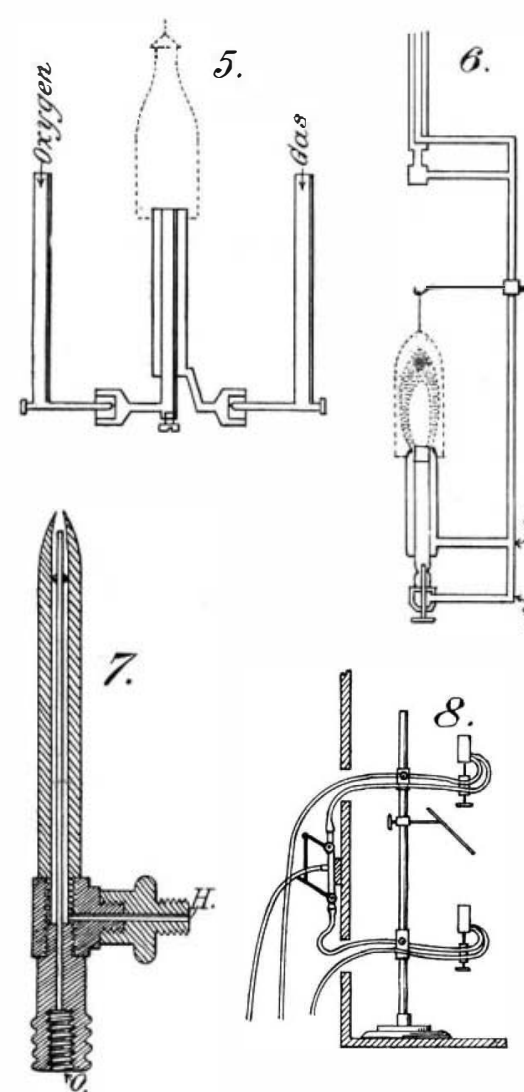

into the barrels. This process was found to be the ly way to save many otherwise valueless beer kegs. introduction of three-fourth to one per cent of oxygen into coal gas before its admission to the purifiers materially assists the purification. It practically does away with the auxiliary hydroxide of iron purifier which served to remove the sulphuretted hydrogen; the lime being alone sufficient to remove all im purities. It also increases the life of the lime and improves the candle-power of the illuminating gas At the Ramsgate Gas Works in England, 10,000 cubi feet of oxy'gen (of 90 per cent purity) are manufactured daily by Brin's process. This is sufficient to purify 1,500,000 cubic feet of coal gas. To sum up, the addition of a small percentage of oxygen results in the following advantages: economy in time, in labor, and in space; simplicity in operation, immunity from nuisance, perfect purification from sulphuretted hydrogen and carbon dioxide, and higher illuminating power. OXYGEN IN THE MANUFACTURE OF WATER GAS (DA USON GAS).

Water gas, which in this country has nearly displaced coal gas, or at least is mixed with the latter, is obtained by the action of steam on red-hot carbon (coke). The latter decomposes the steam, forming hydrogen and carbon dioxide, which combines with more carbon to form inflammable carbon monoxide (CO). Water gas therefore is mainly a mixture of hydrogen and carbon monoxide, the gases being rendered luminous by passing the same through chamber OXYGEN FOR THE PURIFICATION OF ILLUMINATING GAS.

coal gas for lighting purposes. The first burner in which oxygen was successfully used in connection with a gas flame was invented by Nürnberg of Berlin in 1904. In this burner the jacketing tube is provided with an elbow, so that the oxygen meets with som resistance during the passage to the flame and impinges upon the stream of gas at a sharp angle. This not only leads to a more perfect mixing of the gas but also keeps the flame away from that part of the burne burners in which oxygen is used in conjunction with illuminating gas, are those of Hildebrandt, Mewes, Tresenreuter and Hecker. The latter uses a burner in which the two gases came from opposite direction Such lights consume very little illuminating gas and produce less heat than ordinary gas lights, and are more hygienic. They can not contaminate the air as the oxygen is supplied to the flame, and not taken from the air.

Oxygen is also now being used instead of air in various furnace processes. The addition of 5 per cent of oxygen to the blast would reduce the capacity of the blowing engine and would increase the temperature more rapidly above the tuyeres. The escaping gases would be richer in combustibles and therefore better fuel gas. For the production of Bessemer steel the addition of oxygen reduces the time necessary for the operation. The enrichment with oxygen would save about one sixth on the fuel.

An interesting application of oxygen has recently been patented by Messrs. Menne and Zallenkopf of Germany. It consists in a method of perforating masses of metal by fusion and is particularly useful for farming blast-holes, opening of tap-holes, making holes through armor plates, and the like. This is done by connecting one pole of a circuit supplying electric current with the material to be melted through, while the other pole is connected with a pipe through which oxygen is blown. If the pipe is brought in contact with the metal for an instant, while the oxygen simultaneously flows through the pipe, the resulting shortcircuit spark produces sufficient heat to start the fusion and the oxygen perforates the plate in a fraction of a minute. No electric arc is formed, as the current of oxygen prevents this, or blows it out if one should be formed. Another way to perforate the metal is to allow the formation of an electric arc for a few seconds and then allow a stream of oxygen to play on the heated place, whereby the electric arc is destroyed and the metal burnt away.

THE COMPOUND BLOWPIPE AND ITS APPLICATIONS,

The fact that immense heat is obtained when hydrogen burns has been known long ago and has given rise to the invention of a device for drawing advantage therefrom. In the year 1801 Dr. Robert Hare constructed a contrivance which he called an hydrostatic blowpipe, better known as the oxy-hydrogen blowpipe. The quantity of heat evolved when hydrogen burns in oxygen-according to Julius Thomson-is 34,116 calories for each gramme of hydrogen burned. By means of this blowpipe a temperature of 4,000 deg. F. can be obtained. Since a mixture of the two gases is liable to very dangerous explosions, they are kept in separate vessels and only allowed to mix a short distance from the tip of the blowpipe. The typical form of such blowpipe (Fig. 7) consists of an outer tubing, in the center of which a finer iet is longitudinally placed, ending a short distance from the tip. Hydrogen is admitted through the larger tube and ignited, then oxygen is directed through the inner tube. There can be no risk of an explosion if the gases are kept separate until nearly reaching the nozzle; there it will at the worst merely be a sharp "snap," which causes a sudden extinction of the flame. The latter yields but very little light, but on account of its intense heating power, has found many uses in the arts. One of these is its application to the melting and refining of the ores and alloys of platinum, which cannot be fused in an ordinary furnace. A furnace or crucible for this purpose must be constructed of a nearly infusible sub stance, such as calcium oxide or refractory clay. A stream of the burning gases from the blowpipe is forced through an opening in the cover, made of the same refractory material, falling on an interior crucible containing the metal to be melted. While the flame of the oxy-hydrogen blowpipe is but small and itself only slightly luminous, an intense light can be produced by allowing it to fall upon certain substances which can be heated to a high temperature without melting or burning, such as lime, magnesia and other oxides. This fact led to another interesting application of the blowpipe, namely, the oxy-hydrogen light (calcium light or lime light, sideral light, Drummond's (calcium light or lime light, sideral light, Drummond's
light, magnesia light, zirconia light). As far back as 1820 attempts were made to obtain a safe and intense 1820 attempts were made to obtain a safe and intense
light by ignition and combustion of a piece of lime with the aid of the oxy-hydrogen flame. Goldsworthy Gurney has been the first to show that a cylinder of lime could be brought to a state of dazzling brilliancy by projecting the blowpipe flame on the former. How* 
ever, it was Capt. Thomas Drummond who in 1826 first utilized the lime light for practical purposes, by using it for military signals in connection with the trigonometrical survey in Ireland. At first he used oxygen and alcohol vapor (oxy-calcium light, Bude light), but later substituted hydrogen for the latter. Several years passed, however, before the oxy-hydrogen light received general attention and was put to use for the exhibition of dissolving views, for microscopic work, etc. In most cases the cheaper coal gas was substituted for hydrogen, as is still done to-day.

Attempts have been made in Europe to use the blowpipe flame for general lighting purposes. For a time lime light was used in England for lighting the Queen' barracks of the British War Devartment. The burners had been placed 10 feet high, the light being reflected from mirrors. During the Paris Exposition in 186 several buildings were illuminated by oxy-hydrogen lights, and these attracted much attention. Among the buildings thus lighted were the Tuileries and the Hotel de Ville. A year later some streets and square were provided with these lights. Each post was fitte out with five burners, each using 50 liters of hydrogen and 70 liters of oxygen per hour, giving as much light as the old lamps having nine burners and using 170 liters of carbon gas per hour. In 1870 oxy-hydrogen lights were installed at the railroad depot in Vienna. Still, illumination by the blowpipe flame never passe the experimental stage, this being chiefly due to the high cost of oxygen gas. The only other objection that was raised against these lights was that the rad iating material had to be renewed daily, however, thi drawback was overcome later. With an abundant an cheap supply of oxygen as produced by Claude's, Pictet's, Linde's, Hildebrandt's, and similar processes, we may expect that within reasonable time illumination by oxy--hydrogen or oxy-gas lights is sure to come into general favor. At a meeting of the Pacific Coast Gas Association a few years ago, Prof. G. A. Babrick voiced this opinion and told of some experiments he made with oxy-hydrogen lights. One lamp was place 25 feet high at a spot where there was no othe artificial light. The flame was directed against cylinder of lime and reflected from a silver-plate mirror 15 inches in diameter. The night was dark, and still the smallest type of newspaper could be read at a distance of 250 yards. The lamp consume a mixture of 10 cubic feet of illuminating gas and Babrick also thought that calcium light is in many Babrick also thought that calcium light is in man
ways superior to the electric arc, being devoid of ways superior to the electric arc, being devoid of
the disagreeable violet color of the latter. Being exthe disagreeable violet color of the latter. Being ex
ceedingly white, calcium light is well suited for the ceedingly white, calcium light is well suited for the hades and tints of a picture or dress, etc., are plainly discernible as they are by the diffused light of the day. For the same reason lime light can be used for medical purposes, for lighting up body cavities. The intense white color makes it possible to recognize fine color-changes in the reddish mucou membrane, so that diseases can easily be diagnosed. A piece of quicklime is mostly used as radiato for the oxy-hydrogen light; magnesia can be used in stead, but zirconia (zirconium dioxide) excels both, as it compares most favorably with the electric arc The lime is usually in the form of a cylinder (candle pencil), the soft lime gives more light than the hard pencil), the soft lime gives more light than the har
lime, but is inferior in quality and often badly cenlime, but is inferior in quality and often badly cen-
tered. As minute portions of lime become detached and are volatilized from the spot on the lime on which the jet of the burning gases strikes, thus form ing small grooves, it is necessary to expose a fresh surface to the action of the flame. For that purpose the lime is set in a revolving cup or dropped on pin, which has to be turned at intervals of 10 to 15 minutes, the time depending on the pressure of the stant attention. A further disadvantage is that the lime cylinders have to be kept in air-tight tins an that they last only a few hours. The cracking or splintering of the lime pencils has been partially overcome. Crooks recommends the use of dalamite, mineral composed of the carbonates of calcium and magnesium. According to an English patent, the following composition may be used. Magnesia 3 parts, precipitated chalk 6 parts, plaster of Paris 6 parts calcium oxide 1 part, calcium phosphide 1 part, an prepared chalk 6 parts. This mass is subjected to hydraulic pressure and then baked for 3 to 5 hours It does not absorb moisture or carbon dioxide an does not give off gas, injurious to health, during in candescence, or undergo combustion, as does quicklime. It has also been proposed to surround the lime cylinder with a tabular metal shield, Ieaving an elliptical aperture opposite the gas jet. This shiel prevents cracking and also the formation of the whit and impalpable powder often seen inside the lanter it is hardly probable that lime light will ever be used for general lighting purposes, it is well adapted for illumination during brief periods, as required in carrying on important constructions during the darkness of the night, for scenic illumination of the stage were electric light is not available, for the spectroillustrated lectures (Fig. 8), for medical purposes and for military operations in the field; also in emergency ases for photographing where no natural light can be obtained and where magnesium would fail owing to the dense fumes evolved. By far the best radiant body for the oxy-hydrogen flame is zirconia. This with the blowpipe flame by Tessie du Motay (1860) who used oxygen saturated with a solution of napthalene in petroleum. Zirconia, if pure, remains absolutely unchanged at the highest temperature, is permanently brilliant, and does not volatilize. It is used in form of disks (buttons) or very small cylinders; their small size is an advantage in rendering
them a highly concentrated source of light (Fig. 9). They last a long time and are not subject to atmospheric influences. The zirconia buttons as used by Tessie du Montay, Caron, and Linnemann were unTessie du Montay, Caron, and Linnemann were un-
satisfactory. The disks broke from their base and the platinum mounting often started to melt off when it came in contact with the oxy-hydrogen flame. In 1889 Dr. W. Kochs succeeded in overcoming all diffculties. His cylinders were made by "fritting" the small quantity of other substances. These cylinders were porous and yet sufficiently hard to be readily were porous and yet sufficiently hard to be readily
handled. Zirconia disks or cylinders can now be obhandled. Zirconia disks or cylinders can now be ob-
tained in three different qualities: No. 1, for photometric purposes, lasting only 10 hours, but possessing a most intense incandescence; No. 2, for medical pur poses, stereopticons, and the like, lasting 100 hours; and No. 3, for general illuminating purposes, lasting and brilliant as the electric arc, but for certain pur poses the former are even preferable to the latter, as for instance for the spectroscope and for microphotographic purposes. It is most essential for microscopic work for the light to be accurately centered and to remain so. Once set right, the zirconia light will remain unchanged for hours and always in the center of the condensing lens. The electric arc has to
be "fed" from time to time to regulate the distance between the carbon points, while even in the best
lime, pitting or excavation of the surface takes place, which will remove the incandescent point from the focus of the lens. It is of the utmost importance that the burners be constructed properly so that the least amount of gas at the least pressure may produce the greatest amount of heat and light. The chemical union of the gases should take place not further than one-half inch from the tip; the pressure of the oxygen must be 15 times that of the coal gas or hydrogen. Such a burner is not likely to get hot. If the oxygen pressure is not strong enough the flame may beat back and heat up the burner. The tip of the burner should be about one quarter inch from the zirconia (or lime): the angle of incidence being usually 45 degrees. A a piece of smoked glass. Black spots on the radiator indicate unburned oxygen. For scientific purposes the burners designed by Linnemann are the best on account of their accurate adjustments, for illuminating purposes they are too expensive. Those designed by
Wolz are better adapted for such purposes, they consume but eight gallons of oxygen and a like amount of coal gas per hour, are self-regulating, small and parts of hydrogen and one part of oxygen were condensed by means of a "syringe" worked at a strong pressure into a square metal box from which there issued a long jet of very small bore. This jet passed through a thick oak partition in order that the operator might be protected from the danger incidental to an explosion and bursting of the metal box. Thls
was the form of apparatus originally contrived by Clarke and Newman. Later, the safety jets of Gurney, Henning and others were adapted and the gases delivered separately. The so-called mixed-jet burners (Fig. 10), as used in England, in which the gases are mixed in a chamber-packed with wire gauze-before they issue from the orifice of the tip are to be condemned. In the "blow-through jet" (Figs. 11. 12) the oxygen only is under pressure. In this type of
burner, the illuminating gas can be used directly from the service pipe or any convenient source. It also can be used in connection with a saturator. The latter consists of a chamber filled with some porous material-cotton or the like-which is saturated with ether or light gasoline (Ives patent).
The saturator method (oxy-calcium light) is said to The saturator method (oxy-calcium light) is said to be the most economical means of producing lime Acetylene may be substituted for hydrogen or coal gas, but a specially constructed blow jet has to be employed. According to E. H. Amet, acetylene may be used with the ordinary lime light jet, provided the oxygen is mixed with nitrogen and preferably small quantities of carbon dioxide and carbureted hydrogen. He recommends the following mixture: 18,120 parts
of oxygen, 11,868 parts of nitrogen, 6 parts of carbon dioxide, and a like amount of carbureted hydrogen; two parts of this gas mixture to be used with one part of acetylene (Ozo-carbi light). The calcium light has been used more extensively in this than in any other country. It played quite an important part during the great Civil War of 1861 , where it was used for the for signal purposes, lighthouses and during the erection of large buildings. During the construction of the Brooklyn Bridge in 1870 and 1871 twelve lime lights were in constant use. Prior to the introduction of the electric arc, they were used for stage lighting purposes. They were the most popular illuminant for the magic lantern. While the latter was looked upon in Europe as merely an optic toy, it was used in this country for educational purposes. And in spite of the can still be found in every public school in New York city where they are used in connection with stereopticons for illustrated lectures. They may also be found in some of the public parks, where they are put to use during the concerts in the summer, and in winter for illuminating the frozen lakes for the benefit of the skaters. During political campaigns they may be still popular shadow dances; they are used in the form of spot lights for private entertainments, church fairs, etc., and for moving-picture exhibitions where the electric arc is not available. For lighting purposes they are either provided with silver-plated reflectors or, if a small area is to be illuminated, open lights Yre used. The calcium-light outfits as seen in New
York and elsewhere are, however, of the old-fashioned kind and surely in need of improvement. The uses to which these lights are put are so numerous in large cities that it has become a regular industry there to furnish oxygen and hydrogen (coal gas) in separate cylinders. The latter are of 25 or 50 cubic feet capacity, charged to 225 pounds of gas pressure, the tanks being tested to 600 pounds hydraulic pressure. cylinder black. In England the reverse seems to be the case, although for melding purposes oxygen is the case, although for melding purposes oxygen is
put up in black cylinders even in this country. It therefore seems to be a good idea to provide the different tanks and fittings with left- and right-handed threads, so that only the gas for which the cylinders are intended can be pumped in, thus preventing the possibility of any accident in filling. This method has been adopted by the British Oxygen Company in England. The cost of oxygen for calcium lights is usually 10 cents per cubic foot; the gas is in nearly all cases obtained by the chlorate process. Oxygen thus manufactured cannot be made for less than $\$ 18$ per 1,000 cubic feet, the quantity being limited to 500 to 600 cubic feet per hour, using the largest retort. Therefore, oxygen used for general illuminating purposes will never be produced by chemical means. But by the liquefaction of air and its fractional evaporation we have the means of obtaining industrial oxygen in quantities to meet all requirements. By the Linde process 20,000 cubic feet of gas per hour can be produced, using a plant of 900 horse-power. The cost of oxygen as made by Pictet's method is said to be three fourths cent per 1,000 cubic feet, the gas being of 90 per cent purity; by Claude's process the cost of 1.000 cubic feet of oxygen is $13 / 4$ cents. In the United States nearly all liquid-air oxygen seems to be used for welding and like purposes.

From the theoretical standpoint, illumination by the oxy-hydrogen flame can doubtless be regarded as the most rational system, having many advantages over electric light. Its practical use really only depends on the cost of the oxygen and on how readily this can be procured. Prof. Babrick in an address before the Pacific Coast Gas Association in 1903 declared that the United States Liquid Air and Oxygen Company would soon be able to produce commercial
oxygen for 15 cents per 1,000 cubic feet. Using eight cubic feet of illuminating gas and a nearly equal amount of oxygen, a light equal to that of an electric arc consuming one kilowatt per hour can be obtained. The electric arc used for street lighting often does not exceed 1,000 candle-power, while the oxy-hydrogen flame is able to furnish a light of anywhere between 2,000 and 3,000 candle-power. To use Prof. Babrick's figures, an oxy-hydrogen light of over 2,000 candlepower would cost but little more than one half cent per hour, viz:

8 cubic feet of gas at say 50 cents per 1,000 cubic feet $=$.

6 cubic feet of oxygen at say 25 cents per

1,000 cubic feet $=\ldots \ldots \ldots \ldots \ldots \ldots \ldots, 0.15$

Cost of combined gases per hour.... \$0.55 